\title{
Efectos de una intervención para la mejora de las fortalezas psicológicas en Educación Infantil, valorados a través del test del dibujo de la familia
}

\author{
Eugenia Piñero Ruiz, Julián Arense González, Miriam Moñino García \\ y Olivia López Martínez \\ Universidad de Murcia (España)
}

\begin{abstract}
El objetivo de la Psicología Positiva es mejorar la calidad de vida y prevenir la aparición de trastornos mentales y patologías. Su foco de interés es la prevención y desplaza la atención desde la tradicional y exclusiva preocupación por la reparación de los aspectos negativos de nuestra vida, hacia la construcción de cualidades positivas (Seligman y Csikszentmihalyi, 2000), apoyándose en aquellas características psicológicas positivas que todos poseemos en mayor o menor medida. El objetivo de este trabajo es mejorar las fortalezas psicológicas de los alumnos de Educación Infantil a través de la realización de actividades. La muestra está compuesta por 52alumnos de Educación Infantil pertenecientes al segundo ciclo ( $3^{\circ}$ de Educación Infantil) escolarizados en un centro educativo del municipio de Caravaca de la Cruz, al noroeste de Murcia (España). Se llevó a cabo un estudio de las fortalezas y virtudes psicológicas mediante el uso del Test del Dibujo de la Familia (Corman, 1967), con el fin de valorar si existían diferencias significativas en dichas fortalezas antes y después de una intervención para su mejora.
\end{abstract}

Palabras clave: Psicología positiva, infancia, intervención, fortaleza, virtud.

Effects of an intervention to improve the psychological strengths in early childhood education, assessed through the Family Drawing Test. Positive psychology aims to improve the quality of life and prevent the occurrence of mental disorders and pathologies. Focus of interest is prevention and thus avoid traditional and exclusive concern for repairing the negative aspects of life. It looks for the construction of positive qualities (Seligman and Csikszentmihalyi, 2000), in base of the positive psychological features we all have. The objective of this study is to improve the psychological strengths in early childhood education trought the use of activities. Sample is composed by 52 preschool students at 2nd cycle of Pre-primary Education in Caravaca de la Cruz, north west of Murcia (Spain). Strengths and psychological virtues were evaluated though the use of the Family Drawing Test (Corman, 1967), to assess if there were differences or improvements after the intervention.

Keywords: Positive psychology, childhood, intervention, strength, virtue.

Correspondencia: Eugenia Piñero Ruíz. Departamento de Psicología Evolutiva y de la Educación, Facultad de Psicología, Universidad de Murcia, Campus de Espinardo. C.P.: 30100. Murcia (España). E-mail: eugeniapr@um.es 
Si se compara el grado de confort y desarrollo tecnológico que ha alcanzado la humanidad con el grado de insatisfacción, resentimiento y desconfianza entre culturas que se palpa en el planeta, se podría pensar que el mundo se ha vuelto loco, o que, como mínimo, está desajustado (Maaluf, 2009). Cada vez más, la sociedad pone su mirada en la educación como vía para solucionar estas dificultades desde sus orígenes. Sin embargo, en la actualidad la educación no se ajusta totalmente a las expectativas de los ciudadanos. Nadie duda ya de la necesidad de un nuevo paradigma educativo que se adapte al cambio y prepare a las personas para los nuevos retos a los que han de enfrentarse.

Desde el punto de vista de esta necesidad de cambio de paradigma se enmarca también la psicología, que después de muchos años, ha dejado de centrarse en las debilidades del hombre para adoptar un carácter preventivo basado en el desarrollo de las fortalezas humanas (Salanova y Gumbau, 2016). La psicología positiva de Martin Seligman (1999, 2002), tiende la mano a las escuelas para ayudarles a recordar cuál debe ser su principal objetivo, y por tanto el de la sociedad: enseñar a las personas desde sus primeros años de vida a ser felices.

Antes de centrar su interés en el estudio y la enseñanza de la felicidad, Seligman abordó otros aspectos de la psicología, desarrollando entre otras, una teoría sobre la depresión. En 1967 describió el modelo que denominó "indefensión aprendida". Este postula que los individuos expuestos a sucesos aversivos incontrolables aprenden que sus respuestas y las consecuencias de estas son independientes, repercutiendo en déficits cognitivos, emocionales y motivacionales, e interfiriendo posteriormente en otros aprendizajes. En otras palabras, la indefensión aprendida consiste en el aprendizaje de pensamientos y sentimientos de frustración e impotencia ante las adversidades (Overmier y Seligman, 1967; Maier y Seligman, 1976). Este modelo de indefensión aprendida se ha relacionado con la conducta depresiva humana (Seligman, 1975) mostrando la supremacía de los pensamientos sobre las emociones y sentimientos cuando las personas están deprimidas o se sienten tristes. En esos momentos aparecen pensamientos de incapacidad para conseguir un cambio. Seligman fue más allá del modelo expuesto, razonando que, si las personas son susceptibles de aprender a sentirse mal, también lo son de aprender a sentirse bien (Vopel, 2006). De aquí surge una nueva corriente de la psicología que él mismo denomina psicología positiva.

La psicología positiva tiene como objetivo mejorar la calidad de vida y prevenir la aparición de trastornos mentales y patologías. En esta nueva rama se desplaza el interés desde la mera preocupación por la reparación de aquello que resulta negativo en nuestra vida, hacia la construcción de cualidades positivas (Seligman y Csikszentmihalyi, 2000). La corriente se apoya en tres grandes pilares: las emociones positivas (alegría, esperanza, ilusión, agradecimiento, etc.); los rasgos positivos (rasgos 
de personalidad o fortalezas y virtudes personales que nos ayudan a ser mejores y más felices) y las instituciones positivas (familia, educación, sociedad, etc.).

Centrándonos en el segundo de los pilares, los rasgos positivos, Peterson y Seligman (2004) hablan de "fortalezas humanas" para referirse a las características, manifestaciones psicológicas o recursos positivos de la persona. Proponen seis "virtudes generales" que podrían encontrarse en todas las culturas. A su vez cada una de ellas se descompondría en las aludidas fortalezas. Las seis virtudes descritas por estos autores son: sabiduría, coraje, humanidad, justicia, templanza y trascendencia.

Las virtudes son consideradas universales, aunque sus definiciones no coinciden en todas las culturas. Además, en algunas tradiciones predominan unas sobre otras. Sin embargo, en su totalidad, captarían la noción de buen carácter, es decir, de aquellos rasgos positivos de la personalidad que se consideran importantes para alcanzar una buena vida. (Peterson y Seligman, 2004).

Por otro lado, estos mismos autores consideran que el concepto de virtud es demasiado amplio y abstracto y emplean el concepto de fortaleza para referirse a la manifestación psicológica de cada una de esas virtudes. En otras palabras, las fortalezas humanas serían los ingredientes (procesos o mecanismos) psicológicos que definen las virtudes (Giménez, 2010; Giménez, Vázquez, y Hervás, 2010; Vázquez y Hervás, 2008, 2009).

Tabla 1. Virtudes y Fortalezas (Peterson y Seligman, 2004)

\begin{tabular}{cccccc}
\hline Sabiduría & Coraje & Humanidad & Justicia & Templanza & Transcendencia \\
\hline Creatividad, & Valentía & & & Perdón & Capacidad estética \\
Curiosidad & Persistencia & Amor & Ciudadanía & Humildad & Gratitud \\
Apertura & Amabilidad & Equidad & Prudencia & Esperanza \\
$\begin{array}{c}\text { Deseo de aprender } \\
\text { Perspectiva }\end{array}$ & Vitalidad & Inteligencia social & Liderazgo & Autorregulación & Humor \\
Espiritualidad \\
\hline
\end{tabular}

Si la escuela, desde una perspectiva tradicional, se concibe como un lugar para aprender habilidades instrumentales y adquirir conocimientos que más adelante se necesitarán, desde la psicología positiva, la escuela es también el lugar, y el momento, para trabajar el eje fundamental de esta nueva corriente: la felicidad. Es en la escuela donde se debería comenzar a ser felices y a ser sabios, es decir, la escuela, además de transmitir habilidades instrumentales, tiene como tarea básica la de enseñar a vivir, a ser felices y sabios en nuestra vida (Moreno y Gálvez, 2010).

Desde las premisas planteadas, parece evidente que la psicología positiva y la escuela se necesitan. Para que exista un buen entendimiento entre ambas se debería extender el interés en el ámbito educativo desde las tareas más instrumentales a otras más vitales y emocionales (Aritzeta et al., 2016).

La Ley Orgánica de Educación (2006) define la Educación Infantil como la etapa educativa con identidad propia que atiende a niñas y niños desde el nacimiento hasta los seis años de edad. Según los principios pedagógicos de esta ley, en este ciclo se atenderá progresivamente al desarrollo afectivo, al movimiento y los hábitos de control corporal, a las manifestaciones de la comunicación y del lenguaje, a las pautas 
elementales de convivencia y relación social, así como al descubrimiento de las características físicas y sociales del medio en el que viven. Además, se facilitará que niñas y niños elaboren una imagen de sí mismos positiva y equilibrada y adquieran autonomía personal. Los métodos de trabajo se basarán en las experiencias, las actividades y el juego y se aplicarán en un ambiente de afecto y confianza, para potenciar su autoestima e integración social. El desarrollo educativo de estos principios pedagógicos se encuentra muy cercano a lo que la psicología positiva entiende por el desarrollo de las virtudes y fortalezas. La educación, por tanto, debería tener como objetivo su fomento.

Desde este punto de partida, nos planteamos valorar hasta qué punto una intervención concreta centrada en la mejora de las fortalezas psicológicas, podría tener efectos beneficiosos en dichas características. El reto principal es aplicar estas tareas a la etapa de Educación Infantil y sobre todo valorar su efecto.

\section{MÉTODO}

Teniendo en cuenta el marco teórico planteado el objetivo que nos proponemos es analizar el efecto de una intervención educativa para la mejora de las fortalezas psicológicas en Educación Infantil.

\section{Participantes}

La muestra estuvo formada por 52 alumnos de Educación Infantil seleccionados de forma intencional y no probabilística. Estos alumnos pertenecen al segundo ciclo, concretamente a segundo y tercer curso de Educación Infantil y se encuentran escolarizados en un centro educativo del municipio de Caravaca de la Cruz, al noroeste de Murcia. Del total de la muestra, perteneciente a dos aulas de tercer curso de Educación Infantil, 30 alumnos fueron niños (57.7\%) y 22 fueron niñas (42.3\%).

\section{Instrumentos}

Para este estudio se administró el Test del dibujo de la Familia de L. Corman (1961), técnica proyectiva que evalúa fundamentalmente el estado emocional de un niño. La consigna es solicitar al niño que dibuje "una familia". A partir de las características del dibujo realizado se valoran variables sobre el bienestar emocional del menor, características cognitivas y relaciones familiares. En nuestro trabajo nos centramos en aquellas variables que permitían obtener información acerca del bienestar emocional y características cognitivas con el fin de relacionarlas con las fortalezas psicológicas descritas anteriormente. La selección de dichas variables se realizó mediante el acuerdo de jueces expertos, tanto en la técnica del análisis del dibujo de la Familia, como en la teoría de virtudes y fortalezas de Peterson y Seligman (2004).

Del conjunto de variables analizables a través del test del dibujo de la Familia se seleccionaron varias que fueron relacionadas con diferentes virtudes, entre las que 
encontramos las siguientes: adición de elementos externos a la familia, para valorar la creatividad e imaginación (Sabiduría), el uso de líneas curvas para valorar el gusto estético (Trascendencia), la adición del sol en los dibujos para valorar la alegría y el buen humor (Trascendencia), la cercanía de los personajes para valorar la calidad de la comunicación (Humanidad), los tachones y dibujos puntiagudos para valorar la agresividad (Templanza). Para este estudio también se desarrolló una intervención consistente en actividades para el fortalecimiento de las virtudes psicológicas. Estas actividades se diseñaron teniendo en cuenta: la edad de los alumnos, las fortalezas psicológicas y las competencias educativas a trabajar en esta etapa. Dentro de las actividades encontramos la práctica del yoga. El yoga y la psicología positiva comparten muchos objetivos, especialmente aquellos ligados a la gestión de emociones. Algunos de los beneficios que los niños obtienen de su práctica habitual son:

-El desarrollo del conocimiento y conciencia corporal.

-El desarrollo de la fuerza, equilibrio, coordinación, resistencia y flexibilidad.

-Mejora de la postura corporal.

-Incremento de la concentración y la memoria.

-Potenciación de la creatividad y la imaginación.

-Aprendizaje de una correcta respiración.

-Desarrollo de técnicas de relajación.

-Aumento de la autoestima y autoconocimiento.

-Conexión con la espiritualidad y la capacidad de reflexión.

-Toma de conciencia del cuidado del propio cuerpo.

A continuación, se detallan las seis sesiones. Las actividades de yoga están extraídas del libro "Psicología Positiva en Primaria" de Klaus W. Vopel (2006) y adaptadas para Educación Infantil.

Tabla 2. Actividades de Yoga para el fortalecimiento de las virtudes psicológicas (Vopel, 2006)

\begin{tabular}{lc}
\hline \multicolumn{2}{c}{ Virtud: Moderación } \\
\hline $\begin{array}{l}\text { Medio: Pintura } \\
\text { Cuento: El tragasueños. Michael Ende }\end{array}$ & $\begin{array}{l}\text { Yoga } \\
\text { Virtud: Sabiduría y conocimiento }\end{array}$ \\
\hline \multicolumn{3}{c}{ Yoga } \\
Medio: Volcán y pintura & Actividad: Derretirse. \\
\hline \multicolumn{3}{c}{ Virtud: Humanidad } \\
\hline Medio: Viaje imaginario India & Yoga \\
& Actividad: Trabajar juntos \\
\hline \multicolumn{3}{l}{ Medio: Arcilla y pintura } & Yoga \\
Cuento: Estatuas parlantes de Roma & Actividad: El huevo de avestruz \\
\hline \multicolumn{3}{c}{ Virtud: Coraje } \\
\hline $\begin{array}{l}\text { Medio: Sombras chinas } \\
\text { Ópera: Turandot }\end{array}$ & Yoga \\
\hline \multicolumn{2}{c}{ Actividad: La vela } \\
\hline Medio: Cartulinas “Three blessing exercise” Martin Seligman.
\end{tabular}


Los métodos de trabajo se basaron en las experiencias, las actividades y el juego y se aplicaron en un ambiente de afecto y confianza, para potenciar su autoestima e integración social. Las sesiones fueron de 90 minutos, dos días a la semana.

\section{Procedimiento}

Para la recogida de los datos se obtuvo el consentimiento de los padres de los alumnos, por mediación de los responsables de los centros educativos. Una vez obtenido el consentimiento se procedió a la explicación del proyecto a los maestros tutores de cada uno de los grupos de alumnos, y un investigador entrenado en la técnica realizó el test del dibujo de la familia con cada uno de los niños. Se cumplimentaron también las actividades de intervención, repitiendo posteriormente la realización del dibujo de la familia. Posteriormente se procedió a la sistematización de los datos obtenidos y procesamiento informático de los mismos, para su análisis estadístico, consistente en análisis descriptivos de las virtudes psicológicas antes y después de la intervención, la valoración de su posible mejora, tanto en niños como en niñas. Para el análisis de los resultados se empleó el paquete estadístico SPSS versión 16.0. Una vez finalizada esta fase, se realizaron informes para la comunicación de los principales resultados obtenidos a los distintos responsables educativos.

\section{Análisis de datos}

Para el análisis de datos, se elaboraron tablas de contingencia para describir los resultados antes y después de la intervención. Con el fin de comprobar si esta intervención produce un cambio en la respuesta en los niños, se aplicó el test de Mc Nemar aplicando la corrección de continuidad cuando alguna de las frecuencias esperadas fue menor que 5. Se empleó el software SPSS 19.0 para todos los análisis.

\section{RESULTADOS}

A continuación se presentan las puntuaciones del pretest y postest de las variables del test del dibujo de la familia relacionadas con las virtudes para comprobar su posible mejora tras la aplicación del programa de intervención. A la vista de los resultados mostrados en la tabla 2 y en las figuras 1 y 2 , podemos observar que se ha producido un aumento en cuanto al porcentaje de niños y niñas que habían añadido elementos adicionales (plantas, animales, nubes) a los dibujos (43.3\% en los niños y en un $32.8 \%$ en las niñas), y en la presencia de elementos indicativos de alegría y felicidad (un $13.3 \%$ en los niños y un $37.9 \%$ en las niñas). En el caso de la cercanía entre los personajes, se ha observado una disminución en los dibujos de los niños en el postest (de un $76.7 \%$ a un $43.3 \%$ ), siendo esta disminución menos acusada en chicas. En cuanto a la sensibilidad hacia el ambiente, es decir, la representación de figuras con líneas curvas, se 
observa un leve aumento en las situaciones de postest tanto en niñas como en niños, mientras que no se encuentran diferencias entre el pretest y el postest en cuanto a los elementos indicativos de agresividad.

Tabla 3. Porcentaje de aparición de elementos en los dibujos en pretest y postest

\begin{tabular}{lccccccccc}
\hline & \multicolumn{4}{c}{ Niño } & \multicolumn{5}{c}{ Niña } \\
\cline { 2 - 11 } & \multicolumn{3}{c}{ Pretest } & Postest & \multicolumn{3}{c}{ Pretest } & Postest \\
\hline Añade elementos al dibujo & Sí & 11 & $6.70 \%$ & 24 & $80.00 \%$ & 10 & $5.50 \%$ & 18 & $81.80 \%$ \\
(imaginación) & No & 19 & $63.30 \%$ & 6 & $20.00 \%$ & 12 & $4.50 \%$ & 4 & $18.20 \%$ \\
\hline Sensibilidad hacia el & Sí & 4 & $3.30 \%$ & 6 & $20.00 \%$ & 3 & $3.60 \%$ & 8 & $36.40 \%$ \\
ambiente (líneas curvas) & No & 26 & $6.70 \%$ & 24 & $80.00 \%$ & 19 & $6.40 \%$ & 14 & $63.60 \%$ \\
\hline Alegría y felicidad (dibujo & Sí & 5 & $6.70 \%$ & 15 & $50.00 \%$ & 6 & $7.30 \%$ & 15 & $68.20 \%$ \\
sol) & No & 25 & $3.30 \%$ & 15 & $50.00 \%$ & 16 & $2.70 \%$ & 7 & $31.80 \%$ \\
\hline \multirow{2}{*}{ Cercanía entre los personajes } & Sí & 23 & $6.70 \%$ & 13 & $43.30 \%$ & 13 & $9.10 \%$ & 7 & $31.80 \%$ \\
& No & 7 & $3.30 \%$ & 17 & $56.70 \%$ & 9 & $0.90 \%$ & 15 & $68.20 \%$ \\
\hline Elementos indicativos de & Sí & 2 & $0.00 \%$ & 3 & $3.30 \%$ & 6 & $7.30 \%$ & 3 & $3.60 \%$ \\
agresividad & No & 8 & $0.00 \%$ & 7 & $6.70 \%$ & 6 & $2.70 \%$ & 9 & $6.40 \%$ \\
\hline
\end{tabular}

Figura 1. Evolución de los porcentajes de aparición de elementos en los dibujos Pretest Postest Niños

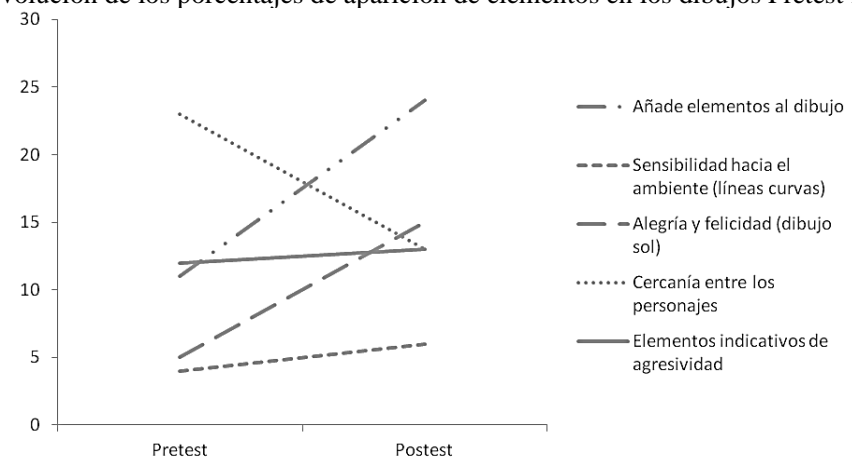

Figura 2. Evolución de los porcentajes de aparición de elementos en los dibujos Pretest Postest Niñas

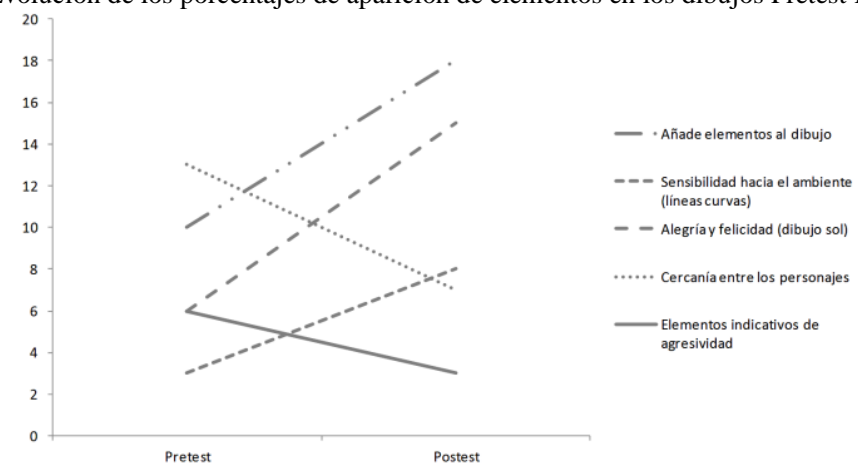

Para comprobar la eficacia de la intervención, se analizaron los cambios de tendencia en cuanto a los porcentajes en cada una de los elementos considerados antes y 
después de la intervención mediante el test de Mc Nemar. En cuanto a la adicción de elementos al dibujo (Tabla 3), de 19 niños que no adicionaron elementos en el primer dibujo, $17(89.5 \%)$ sí los añadieron en el segundo, mientras que 4 que habían añadido este tipo de elementos en el primer dibujo, no lo hicieron en el segundo (66,7\%). Esto muestra una tendencia positiva en cuanto a la adicción de elementos al dibujo, siendo esta diferencia estadísticamente significativa $(p<.001)$. En el caso de las niñas, 9 añadieron elementos en el segundo dibujo de las 12 niñas que no lo habían hecho en el pretest $(75 \%)$, mientras que únicamente un $25 \%$ ( 1 de 4 ) que no lo hizo en el postest lo había hecho en el pretest $(p<.01)$.

Tabla 4. Frecuencia en niños y niñas en la adicción de elementos al dibujo antes y después de la intervención

\begin{tabular}{|c|c|c|c|c|c|}
\hline \multirow{5}{*}{ Niño*** } & & & \multicolumn{2}{|c|}{ Postest } & Total \\
\hline & & & Sí & No & \\
\hline & \multirow{2}{*}{ Pretest } & Sí & 7 & 4 & 11 \\
\hline & & No & 17 & 2 & 19 \\
\hline & \multicolumn{2}{|c|}{ Total } & 24 & 6 & 30 \\
\hline \multirow{5}{*}{ Niña** } & & & \multicolumn{2}{|c|}{ Postest } & Total \\
\hline & & & Sí & No & \\
\hline & \multirow{2}{*}{ Pretest } & Sí & 9 & 1 & 10 \\
\hline & & No & 9 & 3 & 12 \\
\hline & \multicolumn{2}{|c|}{ Total } & 18 & 4 & 22 \\
\hline
\end{tabular}

Test Mc Nemar con corrección de continuidad: * $p<.05$;

$* * p<.01 ; * * * p<.001$

En relación a la sensibilidad hacia el ambiente, y a la presencia de elementos indicativos de agresividad en el dibujo, no se apreció una influencia clara de la intervención en niños y en niñas (Tablas 5y 6).

Tabla 5. Frecuencia en niños y niñas de sensibilidad hacia el ambiente antes y después de la intervención

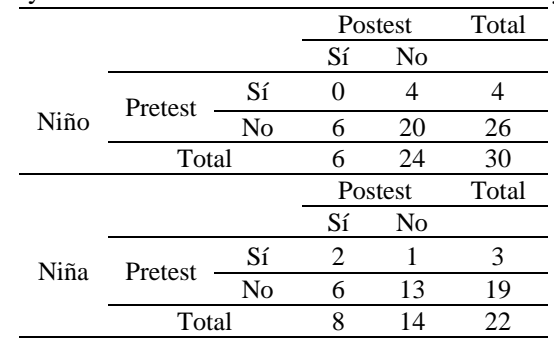

Test Mc Nemar con corrección de continuidad: $* p<.05 ; * * p<.01 ; * * * p<.001$

La intervención sí se mostró efectiva en cuanto a la presencia de elementos indicativos de alegría y felicidad en ambos sexos, mostrando en ambos casos un cambio de tendencia positivo en cuanto a la aparición de estos elementos (Tabla 6). De 25 niños cuyos dibujos no incluían elementos indicativos de alegría y felicidad en el pretest, 14 los hicieron en el postest (56\%), mientras que un $26.7 \%$ de los que no lo hicieron en el postest, lo habían hecho en el primero. En el caso de las niñas, estos porcentajes son de $62.5 \%$ y $14.3 \%$ respectivamente. 
Tabla 6. Frecuencia en niños y niñas de la presencia de elementos indicativos de agresividad en el dibujo antes y después de la intervención

\begin{tabular}{|c|c|c|c|c|c|}
\hline \multirow{5}{*}{ Niño } & & & \multicolumn{2}{|c|}{ Postest } & \multirow{2}{*}{ Total } \\
\hline & & & Sí & No & \\
\hline & \multirow{2}{*}{ Pretest } & Sí & 4 & 8 & 12 \\
\hline & & No & 9 & 9 & 18 \\
\hline & \multicolumn{2}{|c|}{ Total } & 13 & 17 & 30 \\
\hline \multirow{5}{*}{ Niña } & & & \multicolumn{2}{|c|}{ Postest } & \multirow{2}{*}{ Total } \\
\hline & & & Sí & No & \\
\hline & \multirow{2}{*}{ Pretest } & Sí & 1 & 5 & 6 \\
\hline & & No & 2 & 14 & 16 \\
\hline & To & & 3 & 19 & 22 \\
\hline
\end{tabular}

Test Mc Nemar con corrección de continuidad: $* p<.05 ; * * p<.01 ; * * * p<.001$

Tabla 7. Frecuencia en niños y niñas de la presencia de elementos indicativos de alegría y felicidad antes y después de la intervención

\begin{tabular}{|c|c|c|c|c|c|}
\hline \multirow{5}{*}{ Niño* } & & & \multicolumn{2}{|c|}{ Postest } & Total \\
\hline & & & Sí & No & \\
\hline & \multirow{2}{*}{ Pretest } & Sí & 1 & 4 & 5 \\
\hline & & $\mathrm{No}$ & 14 & 11 & 25 \\
\hline & \multicolumn{2}{|c|}{ Total } & 15 & 15 & 30 \\
\hline \multirow{5}{*}{ Niña* } & & & \multicolumn{2}{|c|}{ Postest } & Total \\
\hline & & & Sí & No & \\
\hline & \multirow{2}{*}{ Pretest } & Sí & 5 & 1 & 6 \\
\hline & & No & 10 & 6 & 16 \\
\hline & \multicolumn{2}{|c|}{ Total } & 15 & 7 & 22 \\
\hline
\end{tabular}

$\overline{\text { Test Mc Nemar con corrección de continuidad: * }{ }^{*}<.05 \text {; }}$ $* * p<.01 ; * * * p<.001$

Finalmente, respecto a la cercanía de los personajes (Tabla 7), la intervención pareció influir en los niños $(p<.01)$, no mostrando influencia significativa en las niñas, aunque debemos indicar que estos cambios se produjeron en forma inversa a la esperada.

Tabla 8. Frecuencia en niños y niñas de la cercanía de los personajes en el dibujo antes y después de la intervención

\begin{tabular}{|c|c|c|c|c|c|}
\hline & & & \multicolumn{2}{|c|}{ Postest } & \multirow{2}{*}{ Total } \\
\hline & & & Sí & No & \\
\hline \multirow{3}{*}{ Niño** } & \multirow{2}{*}{ Pretest } & Sí & 10 & 13 & 23 \\
\hline & & No & 3 & 4 & 7 \\
\hline & Total & & 13 & 17 & 30 \\
\hline \multirow{5}{*}{ Niña } & & & \multicolumn{2}{|c|}{ Postest } & \multirow{2}{*}{ Total } \\
\hline & & & Sí & No & \\
\hline & \multirow{2}{*}{ Pretest } & Sí & 3 & 10 & 13 \\
\hline & & No & 4 & 5 & 9 \\
\hline & Total & & 7 & 15 & 22 \\
\hline
\end{tabular}

Test Mc Nemar con corrección de continuidad: $* p<.05$; $* * p<.01 ; * * * p<.001$ 


\section{DISCUSIÓN Y CONCLUSIONES}

El estudio de las virtudes y fortalezas está en sus inicios en todas las etapas del desarrollo humano (Linley et al., 2007; Ramírez, Ortega y Martos, 2015), y muy especialmente en la población infantil y juvenil, por lo que no existen datos que permitan comparar los obtenidos en nuestro estudio, excepto en población adulta en la mayoría de las ocasiones.

Hemos aplicado, además, en nuestro estudio un instrumento que no suele ser el habitual para la medición de virtudes y fortalezas, sino más bien para la evaluación de las relaciones y dinámicas familiares en población infantil. Sin embargo, este instrumento sí ha mostrado ser útil para valorar estos aspectos.

Por estos motivos, se hace compleja la tarea de valorar comparativamente nuestros resultados, y trataremos de elaborar algunas reflexiones sobre ellos.

Hemos comprobado cómo las virtudes psicológicas pueden ser mejoradas y entrenadas en la etapa de educación infantil, con actividades sencillas, adaptadas y que pueden ser aplicadas de forma habitual en las rutinas infantiles. Además, estas actividades inciden en aquellos principios psicopedagógicos que orientan la educación infantil (Méndez y López, 2014).

Observamos mejoras después de la intervención en aquellas fortalezas relacionadas con la creatividad y la imaginación (Virtud de la Sabiduría), el gusto estético (Virtud de la Trascendencia) y la alegría y el buen humor (Virtud de la Trascendencia). Sin embargo, no hemos encontrado diferencias después de la intervención en las virtudes de la Humanidad y en la Templanza, encontrando incluso en cuanto a la Humanidad un cambio inverso al esperado. Estas diferencias en cuanto a la mejora de unas virtudes y no de otras, puede ser debida al diseño de las actividades, centradas sobre todo en la meditación y apreciación de la belleza, más que en la mejora de las habilidades de inteligencia social o relaciones.

Consideramos que es muy importante el trabajo en el estudio y desarrollo de las virtudes y fortalezas desde las edades más tempranas. Por lo general, padres y profesores (y por extensión la sociedad) prestan una especial atención a los comportamientos inadecuados o problemáticos de sus hijos y alumnos. Las sesiones de evaluación en las que participan los niños suelen dirigirse a encontrar problemas o patologías con el fin de subsanarlos de forma temprana, pero se olvida el estudio de aquellos aspectos positivos que podrían mejorar la calidad de vida y felicidad. Es imprescindible prestar más atención a otras medidas que nos permitan conocer el grado de bienestar de la persona. Las diferentes políticas sociales son cada vez más conscientes de esta necesidad y ponen en marcha estudios que recogen datos sobre la satisfacción o la felicidad de los más jóvenes, con el fin de averiguar no sólo la cantidad de felicidad que experimentan, sino qué factores están asociados a ese bienestar. 
Con esta investigación hemos querido hacer una exploración de los posibles cambios producidos en las fortalezas psicológicas del alumnado de Educación Infantil tras una intervención para su mejora. Además consideramos que de esta forma se inicia el camino para conseguir que: la escuela sea un lugar feliz donde se facilite el bienestar de los niños, objetivo básico y central y no secundario; que se desarrollen las fortalezas psicológicas del alumnado, formando niños constructores divertidos, niños que siempre merece la pena observar y escuchar, por interés, curiosidad, pasión, diversión, porque es interesante descubrir qué hace un niño delante de un agujero en una pared o delante de un espejo, o en plena naturaleza; y que el niño aprenda a ser feliz. La felicidad también se aprende (Ellis, 2000). Se aprende a ser feliz y a ser infeliz. Existen actitudes y planteamientos que pueden ser aprendidos. Quizás maestros, familias y sociedad en general, tampoco colaboremos adecuadamente para conseguir dicho objetivo. La escuela ha tenido más una función adaptativa y disciplinar que de desarrollo personal, no es extraño entonces sus dificultades para encontrar el contexto para la transmisión de habilidades no instrumentales.

Los niños absorben como esponjas el clima emocional (López y Sevilla, 2010). Si les mostramos con el ejemplo que alimentamos las emociones positivas, a medida que se vayan desarrollando, crecerán fortaleciéndose y dejando de ser propensos a experimentar emociones negativas y serán ellos mismos generadores de climas emocionales positivos y optimistas.

Es notoria, cada vez más, nuestra preocupación por la mejora de la convivencia en los centros escolares, sobre todo cuando nos asaltan noticias en los medios informativos sobre sucesos producidos por adolescentes o preadolescentes. En este sentido vemos que es necesaria una actuación inmediata. Sin embargo, si realmente se quiere empezar a cambiar algo, quizá sea necesario comenzar desde el principio, y en este caso tendríamos que hablar de prevención. Comenzar desde el principio significaría empezar desde los primeros niveles instruccionales, especialmente desde la etapa de Educación Infantil (López y Sevilla, 2010).

Si la psicología positiva quiere investigar y actuar sobre el desarrollo, la promoción y la prevención de la salud y el bienestar, necesita hacerlo allí donde estos procesos están en plena efervescencia y actuación: la escuela.

\section{REFERENCIAS}

Aritzeta, A., Balluerka, N., Gorostiaga, A., Alonso-Arbiol, I., Haranburu, M., y Gartzia, L. (2016). Classroom emotional intelligence and its relationship with school performance. European Journal of Education and Psychology, 9(1), 1-8.

Corman, L. (1967). El test del dibujo de la familia. Buenos Aires: Kapelusz.

Ellis, A. (2000). Usted puede ser feliz. Barcelona: Paidós. 
Giménez, M. (2010). La medida de las fortalezas psicológicas en adolescentes (VIA-youth): Relación con clima familiar psicopatología y bienestar psicológico. Tesis Doctoral: Universidad Complutense de Madrid.

Giménez, M., Vázquez, C., y Hervás, G. (2010). El análisis de las fortalezas psicológicas en la adolescencia: Más allá de los modelos de vulnerabilidad. Psychology, Society, \& Education, 2(2), 83-100.

Ley Orgánica 2/2006, de 3 de mayo, de Educación. (BOE núm. 106 de 4 de mayo de 2006).

López, O., y Sevilla, A. (2010). Propuesta de un nuevo modelo para estimular el sentido del humor y la creatividad en educación. I Congreso Internacional Virtual de Formación del Profesorado CIFOP. Universidad de Murcia, España.

Linley, P., Maltby, J., Wood, A., Joseph, S., Harrington, S., Peterson, C., Park, N., y Seligman, M. (2007). Character strengths in the United Kingdom: The VIA Inventory of Strengths. Personality and Individual Differences, 43, 341-351.

Maalouf, A. (2009). El desajuste del mundo. Barcelona: Círculo de Lectores.

Maier, S., y Seligman, M. (1976). Learned helplessness: theory and evidence. Journal of experimental Psychology: General, 105, 3-46.

Méndez, M.N.C., y Martínez, O.L. (2014). Psicología Positiva: un camino hacia la práctica educativa en el aula de Educación Infantil. Experiencias de innovación y formación en educación, 131.

Moreno, B., y Gálvez, M. (2010). La Psicología positiva va a la escuela. Típica Boletín Electrónico de Salud Escolar, 1,6.

Overmier, J., y Seligman, M. (1967). Effect of inescapable shock upon subsequent escape and avoidance responding. Journal of Comparative and Phisiological Psychology, 64, 28-33.

Park, N., y Peterson, C. (2006a). Character strengths and happiness among young children: Content analysis of parental descriptions. Journal of Happiness Studies, 7, 323-341.

Peterson, C., y Seligman, M. (2004). Character Strengths and Virtues A Handbok and Classification. Washington, D.C.: APA Press and Oxford University Press.

Ramírez-Fernández, E., Ortega-Martínez, A.R., y Martos-Montes, R. (2015). Las fortalezas en personas mayores como factor que aumenta el bienestar. European Journal of Investigation in Health, Psychology and Education, 5(2), 187-195.

Salanova Soria, M., y Llorens Gumbau, S. (2016). Hacia una psicología positiva aplicada. Papeles del Psicólogo, septiembre, 161-164.

Seligman, M. (1975). Helplessness on depression, development and death. San Francisco: W.H. Freeman.

Seligman, M. (1999). The presidents address. APA. 1998. Annual Report. American Psychologist, $54,559-562$.

Seligman, M. (2002). La auténtica felicidad. Barcelona: Vergara.

Seligman, M. y Csikszentmihalyi, M. (2000). Positive Psychology: an introduction. American Psychologist, 55, 5-14.

Vázquez, C., y Hervás, G. (2008). Psicologia Positiva Aplicada. Bilbao: Desclee de Brouwer.

Vázquez, C., y Hervás, G. (2009) (Eds.). La ciencia del bienestar: Fundamentos de una Psicología Positiva. Madrid: Alianza Editorial.

Vopel, K. (2006). Psicología Positiva en Primaria. Madrid: Editorial CCS.

Recibido: 15 de marzo de 2017 Recepción Modificaciones: 30 de marzo de 2017

Aceptado: 30 de mayo de 2017 